Check for updates

Cite this: Mater. Adv., 2021, 2, 5189

Received 14th May 2021,

Accepted 23rd June 2021

DOI: 10.1039/d1ma00437a

rsc.li/materials-advances

\title{
Bottom-up fabrication of semiconducting 2D coordination nanosheets for versatile bioimaging and photodetecting applications $\dagger$
}

\author{
Arshad Khan $\ddagger$ Shuo Xiang, $\ddagger$ Zeyan Zhou, Chenguang Zhu, Wei Zhang, \\ Guangze Han, Kang Jiang, Dong Li, (D) Anlian Pan (D)* and Qunhong Weng (D)*
}

\begin{abstract}
2D coordination nanosheets (CONASHs) have gained considerable attention due to their highly flexible structures, which favor tailoring band gaps and optical and optoelectrical properties of the materials. The development of this type of 2D materials may generate advanced core materials used in electronic and optoelectronic devices. In this study, for the first time, we report the synthesis of a free-standing CONASH from a two-armed ligand and $\mathrm{Zn}$ via simple liquid-liquid interfacial reactions. The 2D structure was achieved by a rational design through incorporating cyanostilbene functionality into the ligand, which not only assisted in the side-by-side self-assembly of 1D polymers through hydrogen bonds but also endowed excellent photophysical characteristics. The as-synthesized nanosheets exhibited semiconducting properties with an optical band gap of $1.66 \mathrm{eV}$ and strong yellow photoluminescence, which were found to label the lysosomes of mouse 4T1 cells with high brightness, specificity, and photostability. Furthermore, photodetectors fabricated based on the CONASH showed excellent photodetecting properties in a wide visible light region with the detectivity up to $6.09 \times 10^{11}$ Jones and quick responses. This study should motivate the future engineering of diversifying 2D CONASHs based on nonclassical two-arm building blocks with adjustable structural and band properties for broad optical, electrical, and optoelectrical applications.
\end{abstract}

\section{Introduction}

2D materials have numerous unique properties and are compatible with current microelectronic fabrication techniques and standards, thus may have enormous potential to impact the fields of microelectronics, optoelectronics, flexible devices, and so on. ${ }^{1}$ There is a great deal of surge in the development of 2D nanosheets in recent years. For example, as the representative $2 \mathrm{D}$ nanosheet, graphene was found to have excellent charge mobility, mechanical strength, and flexibility, and has been widely explored for scientific and technological applications. ${ }^{2,3}$ However, the zero-band-gap property limits its broad applications, which in turn has stimulated researchers to look out for other alternative 2D nanosheets with open band gaps, such as 2D metal oxides, ${ }^{4-6}$ hexagonal boron nitrides, ${ }^{7-9} 2 \mathrm{D}$ transition-metal chalcogenides (TMDCs), ${ }^{10,11}$ and many others. These new members of the 2D nanosheet family have also exhibited some of the pursuing properties and functionalities, including photoluminescence, ${ }^{4}$

School of Materials Science and Engineering, Hunan University, Changsha, 110016, P. R. China.E-mail: Anlian.pan@hnu.edu.cn,wengqh@hnu.edu.cn

$\dagger$ Electronic supplementary information (ESI) available. See DOI: 10.1039/d1ma00437a \$ These two authors have equally contributed to this manuscript. magnetism, ${ }^{12}$ and semiconductivity. ${ }^{13}$ To prepare the 2D materials, a common strategy is based on the chemical or mechanical exfoliation of their bulk-layered materials, i.e., top-down approach. ${ }^{14,15}$

Among the 2D materials, CONASH is a type of new intriguing member, which has started to attract attention. ${ }^{16,17}$ They are featured by the coordination of alternating organic ligands and metal centres, which can be constructed via either topdown ${ }^{18}$ or a bottom-up strategy. ${ }^{19,20}$ The tremendous library of organic building blocks and available metal ions offers excellent opportunities to tailor the structures and band properties, thereby broadening their applications, particularly in electronic, optical, and optoelectronic fields. The top-down method is featured by its wide applicability in the preparation of various 2D materials but faces the problems of low efficiency, high cost, and difficulty in thickness and size controls for the products. In contrast, the bottom-up strategy provides a more controllable, scalable, and efficient route to construct 2D materials.

In this field, a series of 2D CONASHs have been developed via a bottom-up route with tailor-made functionalities, which were explored for applications in electrochromism, ${ }^{21}$ electrocatalysis, ${ }^{22}$ photoelectric conversions, ${ }^{23}$ and sodium storage. ${ }^{17}$ Nishihara et al. synthesized CONASHs with a redox-modulated electrical 
conductivity property. ${ }^{24}$ Higuchi et al. employed the bottom-up approach and synthesized numerous novel 2D CONASHs with phenanthrolinyl or bipyridyl-metal ion complex structures as potential electrochromic materials, which had short response time, high switching stabilities, and coloration efficiencies. ${ }^{25,26}$ Through designing a porphyrin-Zn CONASH structure, Sakamoto et al. evaluated its photoelectric conversion performances and demonstrated a much-improved photoresponse that covered the whole visible region (400-650 nm) with a high quantum efficiency. ${ }^{27}$ Very recently, Arora et al. fabricated freestanding semiconducting 2D MOF nanosheets $\left[\mathrm{Fe}_{3}(\mathrm{THT})_{2}\left(\mathrm{NH}_{4}\right)_{3}\right]$ via a bottom-up technique, which had high mobility and a small bandgap of $0.45 \mathrm{eV}$ that achieved a broad-band photodetecting covering the wavelength from $400 \mathrm{~nm}$ to $1575 \mathrm{~nm}$. The specific detectivity of the fabricated devices reached up to $7 \times 10^{8}$ Jones with a response time of $\approx 2 \mathrm{~s}$ under the $785 \mathrm{~nm}$ light illumination. $^{28}$ Moreover, Liu and Wöll reported the synthesis of porphyrin-based MOFs by the liquid-phase epitaxial growth, which exhibited superior photophysical properties because of the presence of an indirect bandgap. ${ }^{29}$ Furthermore, some other interesting functionalities of CONASHs containing the bis(dithiolene)metal(II) ${ }^{30}$ and bis(terpyridine)metal(II) ${ }^{31}$ complex motifs were also demonstrated, leading to the design of attractive 2D materials towards important optical, electrical and optoelectrical applications.

In this study, we report the bottom-up synthesis of a novel semiconducting 2Tpy-Zn CONASH via liquid-liquid interface reactions by a two-arm cyanostilbene-containing bisterpyridine ligand and $\mathrm{ZnSO}_{4}$ (Scheme 1). The choice of bisterpyridine (2Tpy) as a coordination fragment is due to its ability to form numerous coordination linkages with a variety of transition metals towards the design of a supramolecular assembly. ${ }^{32-34}$ The introduction of a cyano motif into the coordination ligand not only favors the generation of a first 2D CONASH structure via the $\mathrm{CN} \cdots \mathrm{H}$ hydrogen bond driving the self-assembly but also boosts the photoluminescence properties of the materials. ${ }^{35-37}$ To the best of our knowledge, this strategy of fabricating 2D CONASHs from a two-arm 2Tpy motif by an interphase-assisted "bottom-up" approach is the first example of such design and thus would unlock its potential as a building block towards vast arrays of 2D sheets. The as-prepared CONASHs showed a significantly reduced optical bandgap of $1.66 \mathrm{eV}$ compared with the pristine ligand and exhibited strong and stable yellow photoluminescence with the internal quantum yield of $15.4 \%$. CONASH nanoparticles can target and label the lysosomes of the $4 \mathrm{~T} 1$ cells with high brightness, specificity, and photostability. More interestingly, the sheets were found to have excellent detectivity and sensitivity in the detection of lights across a broad

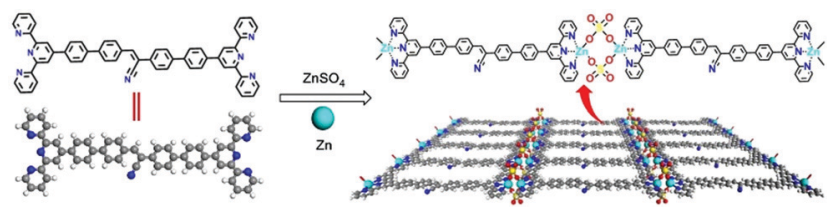

Scheme 1 Chemical structures of 2Tpy ligand and the CONASH of 2Tpy-Zn. visible light region. Such documented optical and optoelectrical properties of the designed CONASHs will intrigue future developments of band-gap adjustable semiconducting 2D materials.

\section{Results and discussion}

The 2Tpy-Zn CONASH was synthesized via a simple interfacial bottom-up growth with thin yellowish membranes obtained at the liquid-liquid interfacial region, which can be transferred onto various substrates for microscopic and spectroscopic investigations. The optical and SEM images of the 2Tpy-Zn CONASH on the Si wafer confirm the formation of a continuous and smooth sheet-like morphology with a lateral size over $0.1 \mathrm{~mm}$ (Fig. 1a and b). The TEM analysis reveals the amorphous feature of the obtained 2Tpy-Zn CONASH (Fig. 1c). Furthermore, the thickness of the sheets on the $\mathrm{SiO}_{2}$ substrate is around $90 \mathrm{~nm}$, as shown in the AFM image and the corresponding height profile (Fig. 1d). Elemental mapping (SEM/ EDS) results in Fig. 1e confirm the presence of $\mathrm{C}, \mathrm{N}, \mathrm{S}, \mathrm{O}$, and $\mathrm{Zn}$ elements, and their homogeneous distributions in the nanosheet except for oxygen. The enriched $\mathrm{O}$ distributions in the sheet edge regions may be caused by the adsorptions of water during the growth.

The coordination structure of the obtained 2Tpy-Zn CONASH was determined by XPS and FTIR spectroscopy. XPS survey (Fig. 2) gives both chemical compositions and bonding states of the nanosheets. The result reveals that the atomic ratios for $\mathrm{Zn}: \mathrm{N}: \mathrm{S}$ is $1: 3.08: 0.76$, which is close to the theoretical stoichiometry for the $\mathrm{SO}_{4}{ }^{2-}$ bridged coordination structure $\left(\mathrm{Zn}-\mathrm{O}-\mathrm{SO}_{2}-\mathrm{O}-\mathrm{Zn}\right)$, as shown in Scheme 1. The binding energy of N1s shifts to a higher value $(399.4 \mathrm{eV})$ in the spectrum compared with the pristine structure (398.7 eV, Fig. S6, ESI $\dagger$ ), suggesting the successful formation of coordination between the Tpy moiety and $\mathrm{Zn}^{2+}$. The shoulder peak at $398.6 \mathrm{eV}$ at $\mathrm{N} 1 \mathrm{~s}$ is originated from the $\mathrm{C} \equiv \mathrm{N}$ moiety in the cyan group. Furthermore, the S2p core level spectrum features the low binding energy at $167.8 \mathrm{eV}$ compared with the $\mathrm{SO}_{4}{ }^{2-}$ moiety that usually appears at $169.0 \mathrm{eV},{ }^{38}$ which is likely caused by the bridging architecture in the nanosheets and is in close agreement with the reported value. ${ }^{31}$ For further clarification, FTIR spectroscopy was performed and the results supported the bridging coordination via the $\mathrm{Zn}_{2}\left(\mu-\mathrm{O}_{2} \mathrm{SO}_{2}\right)_{2}(\mathrm{Tpy})_{2}$ motif in the nanosheet, which exhibited a characteristic vibration peak at $1114 \mathrm{~cm}^{-1}$ assigned to the $\nu_{3}$ mode of $\mathrm{SO}_{4}{ }^{2-}$ with

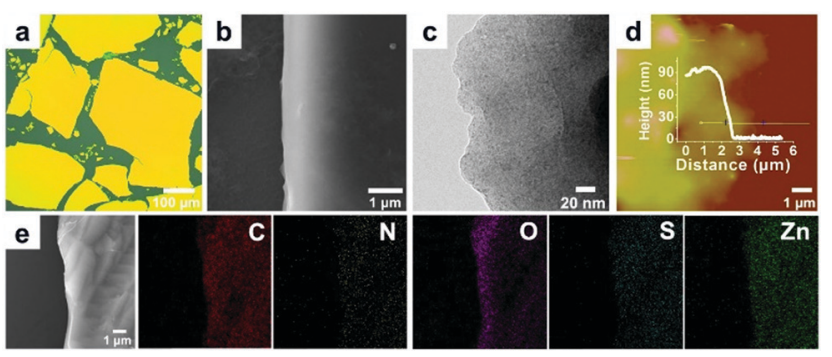

Fig. 1 Structural characterizations of 2Tpy-Zn CONASH. (a) Optical image (b) SEM image, and (c) TEM image of the formed 2Tpy-Zn CONASH. (d) AFM image and height profile of the nanosheet. (e) Elemental mapping of C, N, O, S and Zn elements for the 2Tpy-Zn CONASH. 

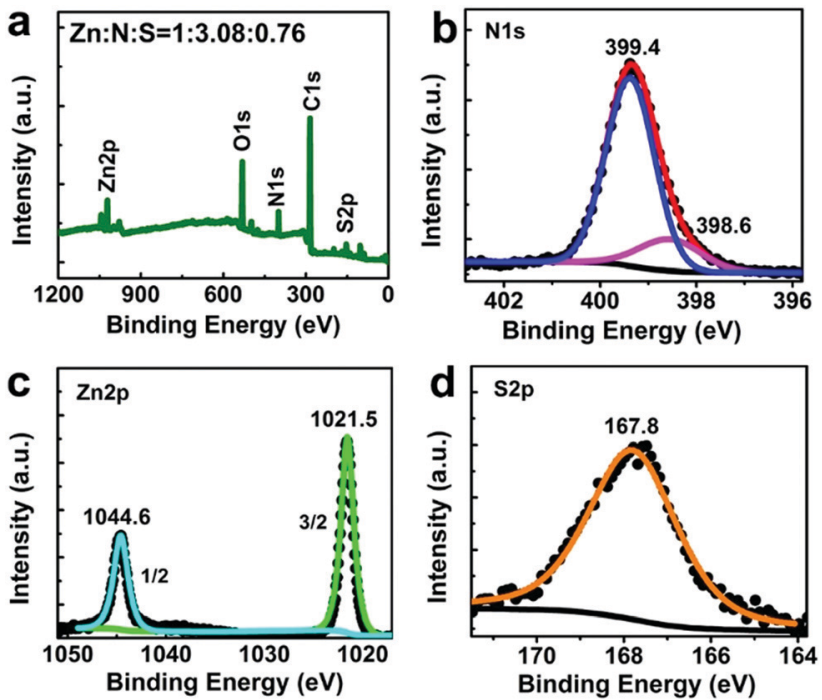

Fig. 2 XPS spectra (a) survey. (b) N1s. (c) Zn2p and (d) S2p core levels.

additional peaks cantered at $1250 \mathrm{~cm}^{-1}$ and $1014 \mathrm{~cm}^{-1}$ arising from the change in the symmetry of $\mathrm{SO}_{4}{ }^{2-}$ structure (see ESI, $\dagger$ Fig. S5). ${ }^{39}$ Such bridging coordination motif of $\mathrm{Zn}_{2}\left(\mu-\mathrm{O}_{2} \mathrm{SO}_{2}\right)_{2}(\mathrm{Tpy})_{2}$ has been also reported in the previous studies. ${ }^{40,41}$

The optical properties of the as-prepared nanosheet were studied via UV-Vis-NIR and photoluminescence spectroscopies on quartz substrates. The yellowish $2 \mathrm{Tpy}-\mathrm{Zn}$ CONASHs formed at the interfacial regions exhibit strong yellow photoluminescence under UV light irradiation (Fig. 3a-d). As shown in Fig. 3e, the 2Tpy ligand has strong absorptions mainly in the UV range with the onset absorption at $\approx 500 \mathrm{~nm}$. After coordination with $\mathrm{Zn}^{2+}$, an enormous red shift of the absorption onset in the 2Tpy-Zn spectrum occurs at $\approx 750 \mathrm{~nm}$. The calculated optical bandgap for CONASH is $1.66 \mathrm{eV}$ (Fig. 3f), which is comparable with those of small-bandgap monolayered transition-metal dichalcogenides
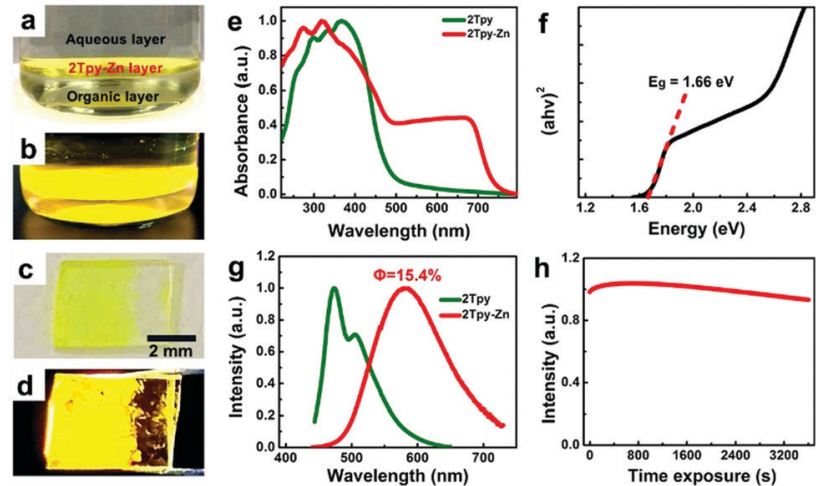

Fig. 3 Optical properties of 2Tpy-Zn CONASH. (a-d) Photographs of the $2 \mathrm{Tpy}-\mathrm{Zn} \mathrm{CONASH}$ thin layer grown at the liquid/liquid interface and transferred to a quartz substrate. (a and c) are under day light, while ( $b$ and d) are taken under UV irradiation. (e) UV-Vis spectra of 2Tpy-Zn nanosheets and 2Tpy ligand. (f) Tauc plot of the CONASH. (g) Photoluminescence spectra of the nanosheets and the pristine ligand. (h) Photostability measurement of the sheet under the irradiation of $\lambda_{\mathrm{ex}}=400 \mathrm{~nm}$ and recorded at a time interval of $10 \mathrm{~s}$.
(TMDCs), such as $\mathrm{MoSe}_{2}(1.57 \mathrm{eV})$ and $\mathrm{WSe}_{2}(1.67 \mathrm{eV}) .{ }^{42}$ This suggests that the coordination of $\mathrm{Zn}^{2+}$ to the 2Tpy ligand is essential for the visible-light absorption properties and remarkably reduced bandgap of CONASH. The observed absorptions in the emerging higher wavelength region of the 2Tpy-Zn CONASH are assignable to the ligand-based intermolecular charge transfer effects, which usually have lower absorbance than the $\sigma-\sigma^{*}$ and $\pi-\pi^{*}$ transitions of the ligand structure. ${ }^{31,40}$

Moreover, the PL emission spectra (Fig. 3g) of the coordinated structure red-shifted from $472 \mathrm{~nm}$ to $589 \mathrm{~nm}$ compared with the pristine ligand, which further signifies the successful accomplishment of the coordination assembly between the ligand and $\mathrm{Zn}^{2+}$. We further investigated the photostability of the nanosheets as a function of irradiation time at $\lambda_{\mathrm{ex}}=400 \mathrm{~nm}$, as shown in Fig. $3 \mathrm{~h}$. The PL intensity has shown negligible intensity loss after exposure to one-hour irradiation, which highlights the remarkable capability of CONASH against photobleaching. This impressive photostability is critical for optoelectrical, bioimaging, and other optic-related applications. The absolute quantum yields $(\Phi)$ of the 2 Tpy-Zn CONASH membrane and aqueous suspension were determined to be $15.4 \%$ and $9.8 \%$, respectively.

The morphology of the 2Tpy-Zn CONASH membrane could be tuned into nanoparticles (NPs) after the sonication treatment of the material in DMSO. TEM image characterization shows that the average particle size is around $12 \mathrm{~nm}$ (Fig. $4 \mathrm{a}$ and b). In order to evaluate the application prospects of $2 \mathrm{Tpy}-\mathrm{Zn}$ in bioimaging, cytotoxicity test was performed by the MTT assay in $4 \mathrm{~T} 1$ cells. The results in Fig. 4c show that after 24 hours of culture at a concentration of $25 \mu \mathrm{g} \mathrm{mL} \mathrm{m}^{-1}$, the cell viability is about $94 \%$; when the concentration was increased to $200 \mu \mathrm{g} \mathrm{mL} \mathrm{m}^{-1}$, the cell viability slightly reduced to about $85 \%$, indicating that $2 \mathrm{Tpy}-\mathrm{Zn}$ has very low cytotoxicity and good biocompatibility. Moreover, COANSH NPs in DMSO exhibit excellent photostability without any prominent intensity changes over one hour, as shown in ESI, $\dagger$ Fig. S7.

Taking advantage of the excellent photoluminescence properties of the designed 2Tpy-Zn CONASH NPs, we explored the
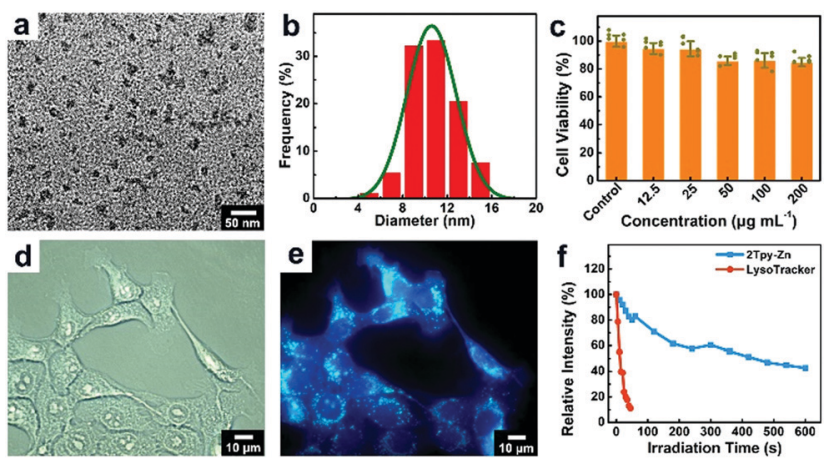

Fig. 4 Live cell imaging of 2Tpy-Zn CONASH NPs. (a) TEM image of 2Tpy-Zn CONASH NPs. (b) Size distribution profile of the nanoparticles. (c) MTT assay of the sample in 4T1 cells for $24 \mathrm{~h}$. (d and e) Bright-field microscopic and corresponding fluorescence $\left(\lambda_{E x}=350 \mathrm{~nm}\right)$ images of the 4T1 cells labelled with CONASH NPs. (f) Photostability tests of 2Tpy-Zn NPS $\left(\lambda_{\mathrm{Ex}}=350 \mathrm{~nm}\right)$ with the comparison of commercially available LysoTracker $\left(\lambda_{\mathrm{Ex}}=540 \mathrm{~nm}\right)$. 
potential applications of the material for live cell imaging. For this purpose, the cells were incubated with 2Tpy-Zn NPs for 30 min. As shown in Fig. 4d and e, CONASH NPs exhibit good capability towards cellular internalizations and the intaken NPs show bright blue fluorescence at UV excitations $\left(\lambda_{\mathrm{Ex}}=350 \mathrm{~nm}\right)$. Even at an ultralow concentration of $1.57 \mu \mathrm{g} \mathrm{mL}^{-1}$, the fluorescence labelling for the cells is still clearly visible, as shown in Fig. S8 (ESI $\dagger$ ) $c_{2}$. This should be attributed to the high quantum yield of the 2Tpy-Zn structure. Moreover, the co-localization experiment shown in Fig. S9 (ESI $\dagger$ ) reveals the clear lysosometargeting and labelling behaviors of 2Tpy-Zn NPs. In addition, under continuous and intensive UV irradiation $\left(\lambda_{\mathrm{Ex}}=350 \mathrm{~nm}\right)$, 2Tpy-Zn NPs show much enhanced photostabilities in live cell imaging, which hold $83 \%$ (after $60 \mathrm{~s}$ ) or $43 \%$ (after $600 \mathrm{~s}$ ) fluorescence intensity compared with only $\approx 10 \%$ retention after $45 \mathrm{~s}$ irradiation for the commercially available LysoTracker (Fig. S10, ESI $\dagger$ ). These findings guide the potential applications of such 2D CONASH NPs for live cell imaging and lysosome labelling. In addition, we also performed the cell imaging of the normal cell line (HEK 293T), and the result shows good imaging capability of CONASH NPs (Fig. S11, ESI $\dagger$ ).

The semiconducting properties of the designed 2Tpy-Zn CONASH make it possible for optoelectrical applications. The CONASH membrane was then employed as the core material for photodetectors; their photoswitching behaviors were tested under the lights/dark cycles at $450 \mathrm{~nm}, 550 \mathrm{~nm}$, and $633 \mathrm{~nm}$ (Fig. 5a-c). Photoresponse is one of the crucial parameters for the photodetectors. Here, very efficient and persistent responses to light are detected without any noticeable deterioration during exposures to the different light irradiations, demonstrating the stable and reproducible performances of the CONASH-based photodetector in the wide wavelength window. The characteristics of current-voltage $(I-V)$ curves (Fig. $5 \mathrm{~d}$ ) at $450 \mathrm{~nm}$ demonstrate the significant enhancement of the photocurrent along with the increase in the illumination intensity. Moreover, the photocurrent measurements at varied voltages show a rapid increase in the generation of current, demonstrating the smooth working operation of the CONASH-based photodetectors (Fig. S12, ESI $\dagger$ ).
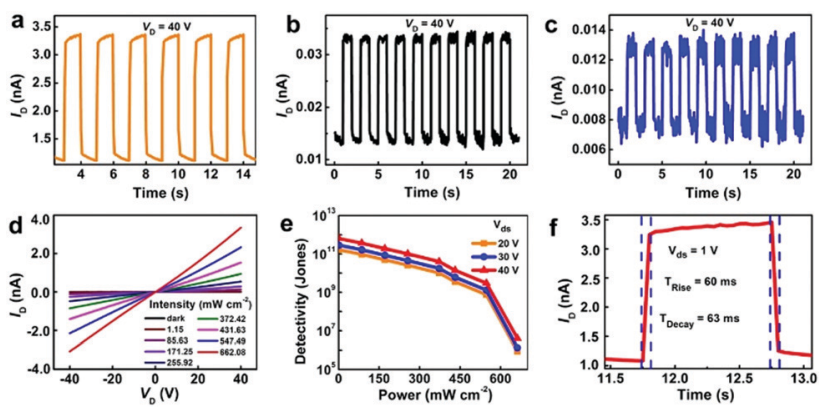

Fig. 5 Photodetecting performances of 2Tpy-Zn CONASH. Photoswitching behavior of the photodetector under irradiations of (a) $450 \mathrm{~nm}$ at $662.08 \mathrm{~mW} \mathrm{~cm}^{-2}$. (b) $550 \mathrm{~nm}$ at $346.96 \mathrm{~mW} \mathrm{~cm}^{-2}$ and (c) $633 \mathrm{~nm}$ at $372.42 \mathrm{~mW} \mathrm{~cm}^{-2}$, respectively. The applied bias $\mathrm{V}_{\mathrm{ds}}$ in $(\mathrm{a}-\mathrm{c})$ are $40 \mathrm{~V}$. (d) $I-V$ profiles at different light power densities of $450 \mathrm{~nm}$ wavelength. (e) Plot of detectivity vs. power density at $450 \mathrm{~nm}$ irradiation. (f) Rise and fall time profile of the photodetector at $450 \mathrm{~nm}$ irradiation.
Besides, responsivity and detectivity are other key parameters for the evaluation of the performances of photodetectors. The maximum responsivity of the device using the equation $R=I_{\mathrm{ph}} /(P \times A)$ was found to be $13 \mathrm{~mA} \mathrm{~W}^{-1}$ at the wavelength of $450 \mathrm{~nm}$ and the bias voltage of $40 \mathrm{~V}$ (Fig. S13, ESI $\dagger$ ). The detectivity was calculated to be $6.09 \times 10^{11}$ Jones at $40 \mathrm{~V}$ (Fig. 5e). As depicted in Fig. 5f, a short rise and decay time of 60 and $63 \mathrm{~ms}$ were recorded, respectively, which implies an outstanding on-off behavior of the fabricated CONASH-based photodetector. In comparison with reported MOF-, COF-, and coordination polymer-based photodetectors, the present photodetector can not only work in a broad spectral region but also shows advantages in detectivity and quick response (Table S1, ESI $\dagger$ ).

\section{Conclusions}

In summary, we have fabricated a novel 2Tpy-Zn CONASH by coordination of a cyanostilbene-functionalized diprotic terpyridine ligand with $\mathrm{Zn}$ ions via a simple liquid-liquid interface reaction. Unlike other CONASHs that are commonly based on three-way coordination modes, the present structure represents the first CONASH constructed by a two-way terpyridine-based motif, opening a new door for $2 \mathrm{D}$ material fabrication. The designed 2Tpy-Zn CONASH exhibited semiconducting properties with an optical band gap of $1.66 \mathrm{eV}$ as well as strong photoluminescence emissions at $589 \mathrm{~nm}$ with a quantum yield of $15.4 \%$. These properties make the 2Tpy-Zn CONASH promising for live-cell imaging and optoelectrical applications. When applied for live-cell imaging, the cytocompatible CONASH NPs have the capability to specifically label the lysosomes of mouse 4T1 cells with bright blue luminescence and high photostability. Besides, the fabricated photodetectors based on the 2Tpy-Zn CONASH can efficiently detect lights across a wide visible light region $(450,550$, and $633 \mathrm{~nm})$ with high detectivity and quick response. This study should inspire novel 2D CONASH designs and future band engineering towards versatile optical, optoelectrical, and biomedical applications.

\section{Experimental section/methods}

\section{Material synthesis}

2Tpy was synthesized by three-step reactions with a total yield of $60 \%$ and was fully characterized via comprehensive analytical methods, including NMR, matrix-assisted laser desorption ionization time-of-flight mass spectrometry (MALDI-TOF MS), FTIR spectroscopy (see details in S1-S5 Sections of ESI $\dagger$ ). The 2Tpy-Zn CONASH was synthesized via a simple bottom-up liquid-liquid interfacial growth, which hosted bilayers of $\mathrm{ZnSO}_{4} \cdot \mathrm{H}_{2} \mathrm{O}(10 \mathrm{mM})$ in water (top layer) and Tpy ligand $(0.1 \mathrm{mM})$ in dichloromethane (bottom layer), respectively, in a $50 \mathrm{~mL}$ beaker at ambient conditions. It is noted that the ligand and zinc salt solutions are needed to be filtered prior to use. The solutions were allowed to stand for one day for the nanosheet growth. The nanosheets were washed thoroughly by water and dichloromethane and transferred onto numerous substrates for tests. 2Tpy-Zn NPs were prepared by 
dispersing the 2Tpy-Zn CONASH layer in DMSO, followed by sonication for $30 \mathrm{~min}$ and collected the supernate by centrifugation at $11000 \mathrm{rpm}$ for $10 \mathrm{~min}$.

\section{Characterizations of materials}

${ }^{1} \mathrm{H}$ NMR spectra were recorded on a Bruker Ascend $600 \mathrm{M}$ instrument $(600 \mathrm{MHz})$ at room temperature using trifluoroacetic acid (TFA) as the solvent and tetramethyl-silane as the reference. Matrix-assisted laser desorption ionization time-of-flight mass (MALDI-TOF) spectrometry was conducted on a AB SCIEX MALDITOF 5800 mass spectrometer. Solid UV-Vis spectra were recorded on a Schimadzu-2600 UV-vis-NIR spectrophotometer. Luminescence excitation and emission spectra were recorded using a FP-8500 fluorescence spectrophotometer (Jasco, Japan) at room temperature by placing samples on quartz plates. SEM coupled with EDS (MIRA3 LMH from TESCAN) was performed for the material morphological and composition analysis. TEM images were recorded on a JEOL JEM 2010 microscope. AFM studies were conducted under the tapping mode on a Veeco Digital Instruments multimode scanning probe microscope with a Nanoscope III controller. FTIR tests were conducted on a Bruker Vertex 70 in the range of $4000-400 \mathrm{~cm}^{-1}$. XPS spectra were recorded on a Thermo Scientific K-Alpha. Optical micrograph and fluorescence images were recorded using a Motic Moticam 2506 and Motic AE31E-EF-INW system, respectively.

\section{Live cell imaging}

The mouse 4T1 cells were sowed on 96-well plates in which the density was controlled to be about $5 \times 10^{3}$ cells per well. When the cells were completely attached, the $2 \mathrm{Tpy}-\mathrm{Zn}$ suspension at different concentrations was added to the cell wells $(10 \mu \mathrm{L}$ per well). After culturing in an incubator for another $30 \mathrm{~min}$, the culture medium in every well was removed and washed with Dulbecco's Phosphate Buffered Saline (DPBS) three times. Subsequently, the 4T1 cells were imaged via fluorescence microscopy. To determine the distribution of 2 Tpy-Zn in cells, a co-localization experiment of $2 \mathrm{Tpy}-\mathrm{Zn}$ and commercial dye (Lyso-Tracker Red, $\lambda_{\mathrm{Ex}}=500-577 \mathrm{~nm}, \lambda_{\mathrm{Em}}=590 \mathrm{~nm}$, Beyotime) were carried out. To evaluate the photostability of the 2Tpy-Zn NPs in cells, the cells were continuously irradiated with a high-power UV ( $\left.\lambda_{\mathrm{Ex}}=350 \mathrm{~nm}, P=150 \mathrm{~W}\right)$ for a certain time interval and analysed the changes in the fluorescence intensity. In addition, the fluorescence intensity change of commercial Lyso-Tracker Red under $540 \mathrm{~nm}$ irradiation $(P=150 \mathrm{~W})$ was also recorded and used for comparisons.

\section{Photodetector fabrications and tests}

The 2Tpy-Zn nanosheets were transferred to quartz substrates for the device fabrication. A photolithographic mask was placed on the substrate and fixed. Next, $10 \mathrm{~nm} \mathrm{Cr}$ and $50 \mathrm{~nm}$ Au were deposited on the material surfaces through thermal evaporations as the source and drain electrodes with the channel length and width of $\approx 477 \mu \mathrm{m}$ and $\approx 50 \mu \mathrm{m}$ length, respectively.

\section{Conflicts of interest}

The authors declare no competing financial interest.

\section{Acknowledgements}

This work was supported by the National Nature Science Foundation of China (NSFC; 21903021), the Innovation Platform and Talent Plan of Hunan Province (2019RS1027), and the Fundamental Research Funds for the Central Universities (Hunan University: 531119200114)

\section{References}

1 Q. Weng, G. Li, X. Feng, K. Nielsch, D. Golberg and O. G. Schmidt, Adv. Mater., 2018, 30, 1801600.

2 K. I. Bolotin, K. J. Sikes, Z. Jiang, M. Klima, G. Fudenberg, J. Hone, P. Kim and H. L. Stormer, Solid State Commun., 2008, 146, 351-355.

3 B. Dlubak, M.-B. Martin, C. Deranlot, B. Servet, S. Xavier, R. Mattana, M. Sprinkle, C. Berger, W. A. De Heer, F. Petroff, A. Anane, P. Seneor and A. Fert, Nat. Phys., 2012, 8, 557-561.

4 H. S. Lee, S.-W. Min, M. K. Park, Y. T. Lee, P. J. Jeon, J. H. Kim, S. Ryu and S. Im, Small, 2012, 8, 3111-3115.

5 T. Sasaki, M. Watanabe, H. Hashizume, H. Yamada and H. Nakazawa, J. Am. Chem. Soc., 1996, 118, 8329-8335.

6 A. Umar and Y. B. Hahn, Nanotechnology, 2006, 17, 2174-2180.

7 W. Lei, D. Portehault, D. Liu, S. Qin and Y. Chen, Nat. Commun., 2013, 4, 1777.

8 T. Sainsbury, A. Satti, P. May, Z. Wang, I. McGovern, Y. K. Gun'ko and J. Coleman, J. Am. Chem. Soc., 2012, 134, 18758-18771.

9 Y. Lin, T. V. Williams and J. W. Connell, J. Phys. Chem. Lett., 2010, 1, 277-283.

10 A. Splendiani, L. Sun, Y. Zhang, T. Li, J. Kim, C.-Y. Chim, G. Galli and F. Wang, Nano Lett., 2010, 10, 1271-1275.

11 Z. Zeng, Z. Yin, X. Huang, H. Li, Q. He, G. Lu, F. Boey and H. Zhang, Angew. Chem., Int. Ed., 2011, 50, 11093-11097.

12 H. Okamoto, Y. Kumai, Y. Sugiyama, T. Mitsuoka, K. Nakanishi, T. Ohta, H. Nozaki, S. Yamaguchi, S. Shirai and H. Nakano, J. Am. Chem. Soc., 2010, 132, 2710-2718.

13 N. Sakai, Y. Ebina, K. Takada and T. Sasaki, J. Am. Chem. Soc., 2004, 126, 5851-5858.

14 J. N. Coleman, M. Lotya, A. O’Neill, S. D. Bergin, P. J. King, U. Khan, K. Young, A. Gaucher, S. De, R. J. Smith, I. V. Shvets, S. K. Arora, G. Stanton, H.-Y. Kim, K. Lee, G. T. Kim, G. S. Duesberg, T. Hallam, J. J. Boland, J. J. Wang, J. F. Donegan, J. C. Grunlan, G. Moriarty, A. Shmeliov, R. J. Nicholls, J. M. Perkins, E. M. Grieveson, K. Theuwissen, D. W. McComb, P. D. Nellist and V. Nicolosi, Science, 2011, 331, 568-571.

15 K. R. Paton, E. Varrla, C. Backes, R. J. Smith, U. Khan, A. O’Neill, C. Boland, M. Lotya, O. M. Istrate, P. King, T. Higgins, S. Barwich, P. May, P. Puczkarski, I. Ahmed, M. Moebius, H. Pettersson, E. Long, J. Coelho, S. E. O’Brien, E. K. McGuire, B. M. Sanchez, G. S. Duesberg, N. McEvoy, T. J. Pennycook, C. Downing, A. Crossley, V. Nicolosi and J. N. Coleman, Nat. Mater., 2014, 13, 624-630.

16 X. Huang, P. Sheng, Z. Tu, F. Zhang, J. Wang, H. Geng, Y. Zou, C.-A. Di, Y. Yi, Y. Sun, W. Xu and D. Zhu, Nat. Commun., 2015, 6, 7408. 
17 S. Liu, J. Zhou and H. Song, Adv. Energy Mater., 2018, 8, 1800569.

18 T. Singha Mahapatra, A. Dey, H. Singh, S. S. Hossain, A. K. Mandal and A. Das, Chem. Sci., 2020, 11, 1032-1042.

19 R. Dong, M. Pfeffermann, H. Liang, Z. Zheng, X. Zhu, J. Zhang and X. Feng, Angew. Chem., Int. Ed., 2015, 54, 12058-12063.

20 T. Kambe, R. Sakamoto, T. Kusamoto, T. Pal, N. Fukui, K. Hoshiko, T. Shimojima, Z. Wang, T. Hirahara, K. Ishizaka, S. Hasegawa, F. Liu and H. Nishihara, J. Am. Chem. Soc., 2014, 136, 14357-14360.

21 K. Takada, R. Sakamoto, S.-T. Yi, S. Katagiri, T. Kambe and H. Nishihara, J. Am. Chem. Soc., 2015, 137, 4681-4689.

22 X. Sun, K.-H. Wu, R. Sakamoto, T. Kusamoto, H. Maeda, X. Ni, W. Jiang, F. Liu, S. Sasaki, H. Masunaga and H. Nishihara, Chem. Sci., 2017, 8, 8078-8085.

23 R. Sakamoto, K. Hoshiko, Q. Liu, T. Yagi, T. Nagayama, S. Kusaka, M. Tsuchiya, Y. Kitagawa, W.-Y. Wong and H. Nishihara, Nat. Commun., 2015, 6, 6713.

24 T. Kambe, R. Sakamoto, K. Hoshiko, K. Takada, M. Miyachi, J.-H. Ryu, S. Sasaki, J. Kim, K. Nakazato, M. Takata and H. Nishihara, J. Am. Chem. Soc., 2013, 135, 2462-2465.

25 M. K. Bera, T. Mori, T. Yoshida, K. Ariga and M. Higuchi, ACS Appl. Mater. Interfaces, 2019, 11, 11893-11903.

26 S. Mondal, Y. Ninomiya, T. Yoshida, T. Mori, M. K. Bera, K. Ariga and M. Higuchi, ACS Appl. Mater. Interfaces, 2020, 12, 31896-31903.

27 R. Sakamoto, T. Yagi, K. Hoshiko, S. Kusaka, R. Matsuoka, H. Maeda, Z. Liu, Q. Liu, W.-Y. Wong and H. Nishihara, Angew. Chem., Int. Ed., 2017, 56, 3526-3530.

28 H. Arora, R. Dong, T. Venanzi, J. Zscharschuch, H. Schneider, M. Helm, X. Feng, E. Cánovas and A. Erbe, Adv. Mater., 2020, 32, 1907063.

29 J. Liu, W. Zhou, J. Liu, I. Howard, G. Kilibarda, S. Schlabach, D. Coupry, M. Addicoat, S. Yoneda, Y. Tsutsui, T. Sakurai,
S. Seki, Z. Wang, P. Lindemann, E. Redel, T. Heine and C. Wöll, Angew. Chem., Int. Ed., 2015, 54, 7441-7445.

30 A. J. Clough, J. M. Skelton, C. A. Downes, A. A. de la Rosa, J. W. Yoo, A. Walsh, B. C. Melot and S. C. Marinescu, J. Am. Chem. Soc., 2017, 139, 10863-10867.

31 T. Tsukamoto, K. Takada, R. Sakamoto, R. Matsuoka, R. Toyoda, H. Maeda, T. Yagi, M. Nishikawa, N. Shinjo, S. Amano, T. Iokawa, N. Ishibashi, T. Oi, K. Kanayama, R. Kinugawa, Y. Koda, T. Komura, S. Nakajima, R. Fukuyama, N. Fuse, M. Mizui, M. Miyasaki, Y. Yamashita, K. Yamada, W. Zhang, R. Han, W. Liu, T. Tsubomura and H. Nishihara, J. Am. Chem. Soc., 2017, 139, 5359-5366.

32 A. Sil, A. Maity, D. Giri and S. K. Patra, Sens. Actuators, B, 2016, 226, 403-411.

33 S. Chakraborty and G. R. Newkome, Chem. Soc. Rev., 2018, 47, 3991-4016.

34 F. S. Han, M. Higuchi and D. G. Kurth, J. Am. Chem. Soc., 2008, 130, 2073-2081.

35 T. Dünnebacke, K. K. Kartha, J. M. Wiest, R. Q. Albuquerque and G. Fernández, Chem. Sci., 2020, 10405-10413.

36 Y. Zhang, J. Sun, X. Lv, M. Ouyang, F. Cao, G. Pan, L. Pan, G. Fan, W. Yu, C. He, S. Zheng, F. Zhang, W. Wang and C. Zhang, CrystEngComm, 2013, 15, 8998-9002.

37 K. A. N. Upamali, L. A. Estrada, P. K. De, X. Cai, J. A. Krause and D. C. Neckers, Langmuir, 2011, 27, 1573-1580.

38 V. Hayez, A. Franquet, A. Hubin and H. Terryn, Surf. Interface Anal., 2004, 36, 876-879.

39 G. Tamasi and R. Cini, Dalton Trans., 2003, 2928-2936.

40 Z. Ma, Y. Cao, Q. Li, M. F. C. Guedes da Silva, J. J. R. Fraústo da Silva and A. J. L. Pombeiro, J. Inorg. Biochem., 2010, 104, 704-711.

41 M. Harvey, S. Baggio, S. Russi and R. Baggio, Acta Crystallogr., Sect. C: Cryst. Struct. Commun., 2003, 59, m171-m174.

42 J. Gusakova, X. Wang, L. L. Shiau, A. Krivosheeva, V. Shaposhnikov, V. Borisenko, V. Gusakov and B. K. Tay, Phys. Status Solidi A, 2017, 214, 1700218. 